\title{
An Open Embedded Hardware and Software Architecture Applied to Industrial Robot Control
}

\author{
Lin Zhang, Peter Slaets and Herman Bruyninckx \\ Department of Mechanical Engineering \\ Katholieke Universiteit Leuven, Belgium \\ \{lin.zhang, peter.slaets, herman.bruyninckx\}@mech.kuleuven.be
}

\begin{abstract}
This paper presents an open embedded hardware and software architecture for industrial robot control. Open hardware refers to open source IP-core provided by the authors, while open software implies the use of open source software, specifically Linux and OROCOS [1]. Embedded implies integration of various functional blocks on one System-On-Chip board, for data acquisition, computation and control tasks, of relatively small size and low power consumption, compared to regular PC-based industrial controllers.

The proposed architecture is a hardware and software codesign where an operating system running on an embedded processor drives the FPGA hardware controllers. It is industrial robot type independent, as long as the motors are equipped with incremental position encoders and driven by PWM signals. The architecture has been tested on an industrial Performer MK2 robot setup. Low cost, low power consumption, remarkable stability, high flexibility and expandability are the key advantages of the architecture for industrial robot control.
\end{abstract}

Index Terms-FPGA, hardware and software architecture, industrial robot control.

\section{INTRODUCTION}

In recent decades, industrial robot control system have evolved from open-loop, position controlled to closed loop, adaptive controlled systems [2]-[4]. The next generation of systems needs to address fast real-time response to cope with unpredictable human interaction, hazard safety issues, as well as increasing process speed demands. Currently, closed loop hardware systems are mainly dominated by microprocessors and digital ICs such as DSPs and RISC, delivering high-speed performance combined with the required flexibility inherent to all programmable solutions [5]. Subsequent advantages of DSP-based robot control systems where denoted by five main features [6]: rapid development cycle, high bandwidth to cost ratio, compact design, modular and upward compatibility, plus open architecture using ANSI C. However, lack of standardization remains a major disadvantage of DSP or ICs. In terms of hardware, there are significant variations in bus compatibility, chip and board design, as well as final system integration. In terms of software development and system migration, recent technological progress in the field of very large scale integration (VLSI) technology and electronic design automation (EDA) techniques has created opportunities for development of more complex high-performance hardware platforms in industrial control.
Flexible hardware such as field programmable gate array (FPGA) is currently considered an appropriate solution to increase control performance [7]. Due to its reprogrammability, FPGA technology combines low cost development with a rising integration density [8] making it well highly suitable for embedded systems.

In machine control applications domain [6], [9], a growing demand in data throughput arises. FPGA technology is successfully implemented in many electric applications such as pulse-width-modulation (PWM) invertors, induction machine drives, motion controls [10], neural networks control, as well as velocity measurements [11], [12]. The success is a natural consequence of cost reduction, low power consumption, reliability, real-time characteristic and significant control performance improvement by dedicated parallel computations.

Current commercial industrial robot controllers [13], [14] are based on modular hardware architectures, consisting of a central processor behaving as a coordinator and various additional co-processors performing low level motor control. These industrial architectures are designed to ensure robot reliability and maintainability. The bound commercial industrial software is usually integrated in the hardware platform, and focuses on position based motion control applications; in addition, lack of extendibility with alternative sensors or axis makes it difficult, or even impossible to extend the functionality of the robot. To address the require of complicated motion control, as well as implementing control algorithm, open source robot control software alternatives [1], [4], [15] are being developed, maintained and widely used. However, open hardware configuration is still in an initial stage.

This paper presents an open embedded hardware and software controller architecture applied to an industrial robot platform. This architecture can be easily extended to control a general multi-axis robot, as long as the motors are equipped with incremental position decoders and driven by PWM signals.

\section{EMBEDDED HARDWARE AND SOFTWARE ARCHITECTURE}

The proposed architecture in Fig. 1 consists of four parts: an industrial robot, a power control bridge, a terminal inter- 


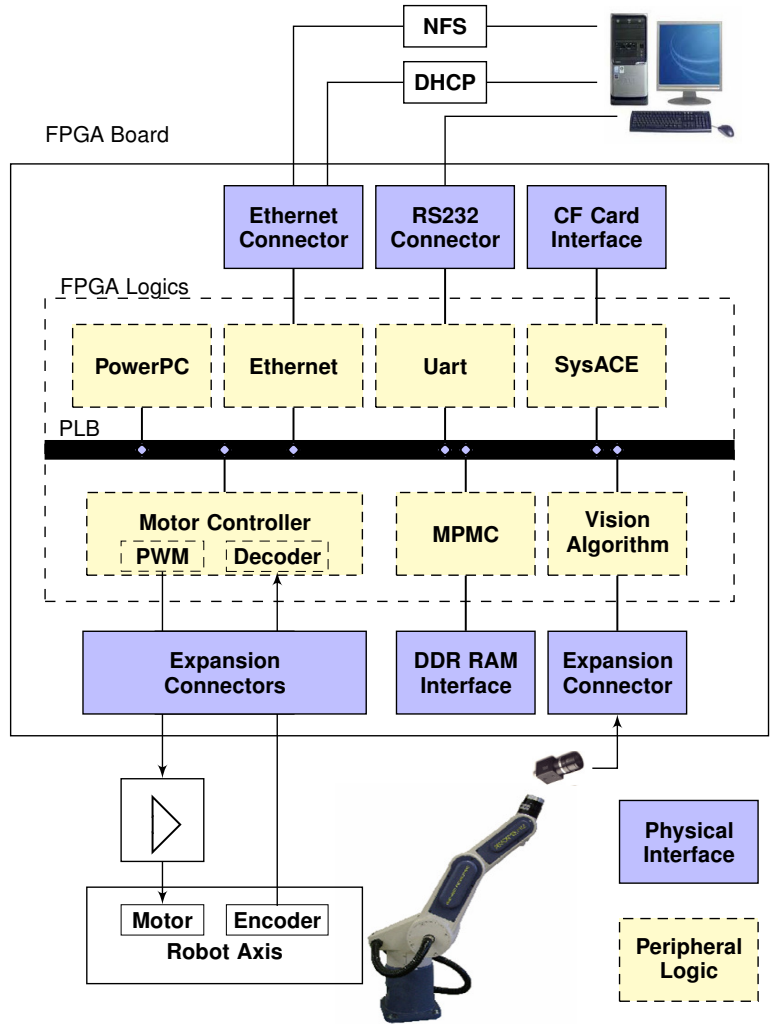

Fig. 1. The embedded robot control architecture consists of four parts: an industrial robot, a power control bridge, a terminal interface and a Systemon-Chip board.

face and a System-on-Chip board. The board consists of an embedded processor running real-time operating system, and an FPGA chip implementing quadrature decoder, control loop and additional functions such as vision algorithm, to relieve the embedded processor from significant computational load [16].

The hardware remains fully flexible due to the reconfigurable characteristic and hardware-software co-design. The FPGA platform assures the extendibility using open-source hardware IP cores, consequently result in a reusable and platform independent open hardware. The terminal is an interface which is accessible from any laptop or PC through a serial port.

In the current setup, a performer MK-2 industrial robot arm is driven by a Xilinx Virtex-II pro board with two integrated PowerPC processors. One of the PowerPC processors runs a real-time Linux and an Open RObot COntrol Software (OROCOS). The software system, consisting of the Linux kernel and hardware configuration for the FPGA, is stored in an ACE-file and is downloaded from a compact flash at boot up. The file system can also be stored on the same compact flash, or alternatively on a remote PC using a Network File System (NFS). The PowerPC is connected to the FPGA via a processor local bus (PLB). Commercial MD10C NMOS Hbridge motor drivers, designed by Cytron Technologies, are used to drive the motors in the current setup. The MD10C supports up to $10 \mathrm{kHz}$ PWM signal for DC motor control.

\section{INCREMENTAL OPTICAL DECODER INTERFACE}

The Performer MK2 industrial robot has five axes. Each axis is driven by a motor, which is equipped with an incremental optical encoder, or a.k.a quadrature encoder. The two quadrature output signals $A$ and $B$ have a 90 degrees phase difference. By measuring $A, B$ and an additional index signal $I$ that generates a pulse every revolution, a relative rotational position can be determined. Fig. 2 is a schematic of the decoder interface, which consists of seven blocks: three input noise filters, a quadrature decoder, a position calculator, a velocity calculator and an acceleration calculator.

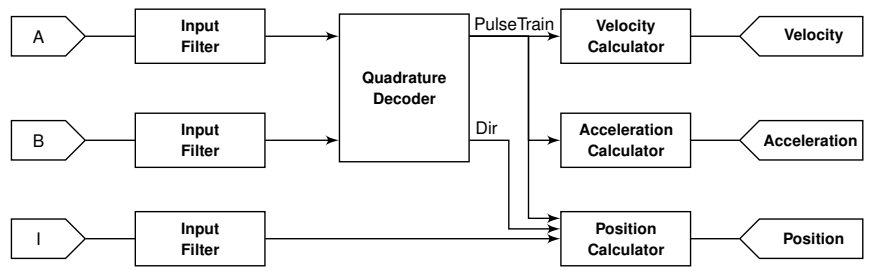

Fig. 2. A schematic of the decoder interface consists of seven blocks: three input noise filters, a quadrature decoder, a position calculator, a velocity calculator and an acceleration calculator.

\section{A. Input filters}

The filters perform signal conditioning which eliminate high frequency noise present in the input signals as shown in Fig. 3. Every digital input value is passed through four D-Flip-Flops (D-FF) in four consecutive clock cycles. The output value is only adjusted when the latter three D-FF outputs coincide. The filter operates properly when the clock frequency is at least two times higher than the decoder capturing frequency, in the current setup, $70 \mathrm{kHz}$. The filter, however, introduces a delay of four clock cycle; but this delay can be reduced by a relatively high sampling frequency e.g. $10 \mathrm{MHz}$.

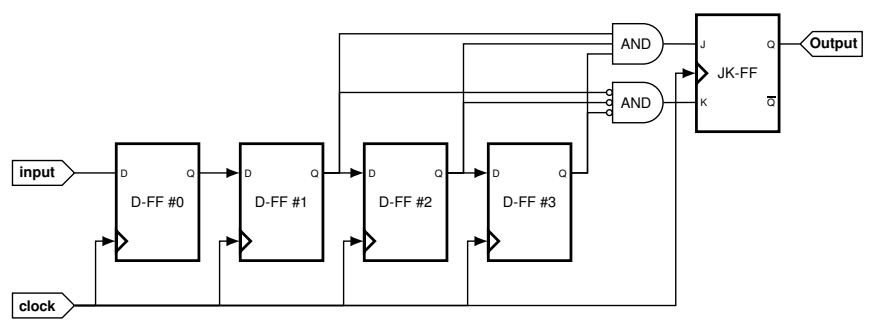

Fig. 3. The input filter consists of four D-flip-flops and a JK-flip-flop. If the latter three D-FFs have the same output values, the output will be updated. 


\section{B. Quadrature decoder}

The filtered signals $A$ and $B$ are decoded to a pulse train signal (PulseTrain) and a rotational direction signal (Dir) by the quadrature decoder. The two decoded signals are used to determine the current position, velocity and acceleration of a motor or a robot axis. Based on [12], the state diagram shown in Fig. 4 is implemented in VHDL.

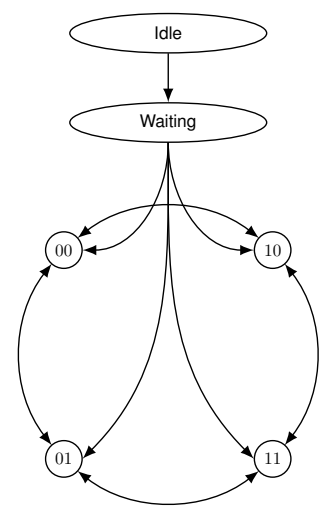

Fig. 4. The quadrature decoder state machine implemented in VHDL.

The state machine starts from Idle state. This state is followed by a Waiting state where the state machine waits for valid input signals $A$ and $B$. The next state is determined by both $A$ and $B$, in a combination of $00,01,10,11$. The PulseTrain and Dir signals are generated due to the change of $A$ and $B$, as demonstrated in Fig. 5 .

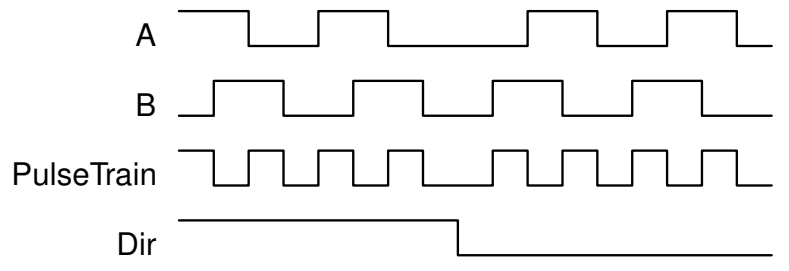

Fig. 5. The PulseTrain and Dir signals are generated due to the change of $A$ and $B$.

\section{Position calculator}

The position calculator calculates the current position based on the number of complete revolution and rotated angle, which are determined by a revolution counter and an angle counter, as shown in Fig. 6. The position is calculated by:

$$
\text { Position }=\frac{(\text { rev } \cdot m+\text { angle }) \cdot \theta}{n}
$$

where $m, \theta$ and $n$ are robot dependent parameters. In the equation, $m$ denotes the number of pulses for each revolution, $\theta$ denotes the angle corresponding to a state shift of the quadrature decoder, and $n$ corresponds to the transmission ratio between the motor and the axis. A similar circuit has been designed by [17].

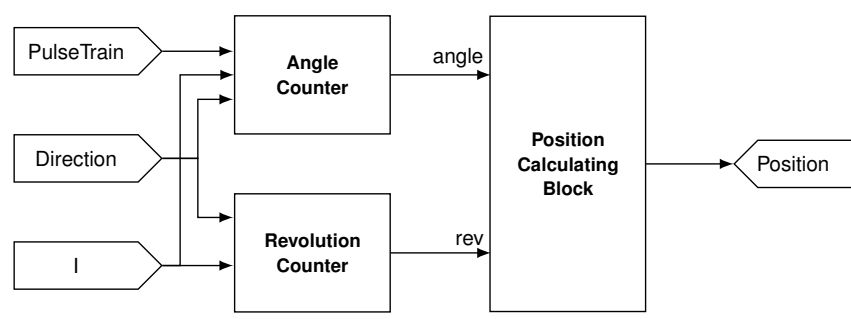

Fig. 6. The position calculator consists of an angle counter, a revolution counter and a position calculating block.

\section{Velocity calculator}

Although analog tachometers are widely used in industry to improve the dynamic performance in a control loop, in [11] several reasons are given to motivate the use of digital tachometers:

- an improved accuracy with less than $2 \%$ relative error;

- no need for $\mathrm{A} / \mathrm{D}$ converter or analog noise filters.

Different techniques have been proposed to obtain reasonable accuracy and response time. Constant elapsed time method, proposed by [18], measures the elapsed time between a variable number of pulses $k$. To assure the accuracy, $k$ is updated in every measurement interval. In this paper, the number of pulses in PulseTrain is counted in a fixed period of time to calculate the precise velocity. However, this method guarantees accuracy only in low- and medium-velocity range [11]. In high velocity range, pulse count method, has a better performance. Both methods are implemented in VHDL.

1) Constant elapsed time method: An N-bit counter is enabled by a pulse and is stopped when a maximum number of pulses $K_{-}$out, determined by the up counter, is reached. Define $f_{b}$ as the input frequency to the counter, and $C_{-}$value as the pre-defined maximum number of pulses, the angular velocity $\omega_{t c}$ of an axis is determined by:

$$
\omega_{t c}=\frac{2 \pi f_{b}}{n} \frac{K_{-} \text {out }}{C_{\_} \text {value }} .
$$

where $n$ denotes the transmission ratio between the motor and the axis. The $C_{-}$value is forwarded to a $K_{-}$calculator to adjust K_out. This mechanism assures a dynamically generated K_out to maintain the accuracy of the results within a certain level, while preserving the tachometer time response, as demonstrated in Fig. 7

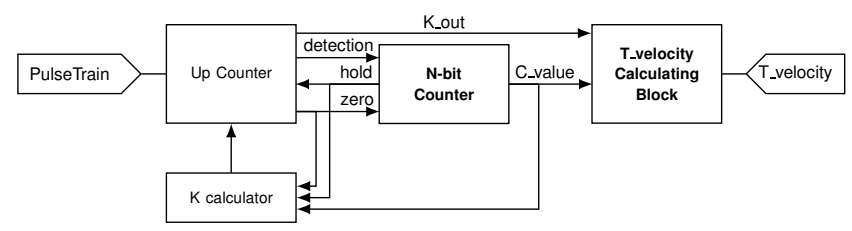

Fig. 7. The constant elapsed time velocity calculator. 
2) Pulse count method: In a fixed time interval $T_{c}$, the decoder pulses are counted and stored in PT_C_value. The angular velocity $\omega_{p c}$ is determined by P_velocity Calculating Block in Fig. 8 .

$$
\omega_{p c}=\frac{2 \pi f_{c}}{n} \frac{P T_{\_} C_{-} v a l u e}{m}
$$

with $f_{c}=\frac{1}{T_{c}}, m$ is the number of pulses for each revolution, and $n$ is the transmission ratio between the motor and the axis.

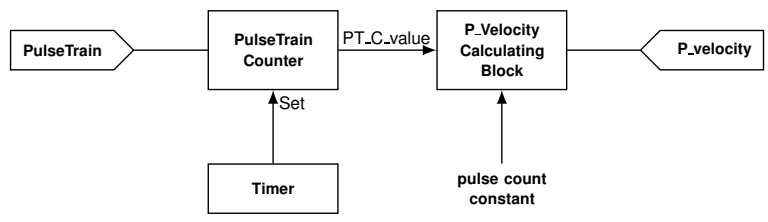

Fig. 8 . The pulse count velocity calculator.

\section{E. Acceleration calculator}

The acceleration calculator calculates the acceleration based solidly on the decoder pulses, as shown in Fig. 9 . The principle is derived from the pulse count method where for a time period $T_{c}$, the incoming pulses are counted by a PulseTrain counter and loaded into a shift register. The acceleration is determined by:

$$
a=\frac{2 \pi}{n T_{c}^{2}} \frac{\text { current }- \text { previous }}{m}
$$

where $n$ denotes the transmission ratio between the motor and the axis, and $m$ denotes the number of pulses for each revolution.

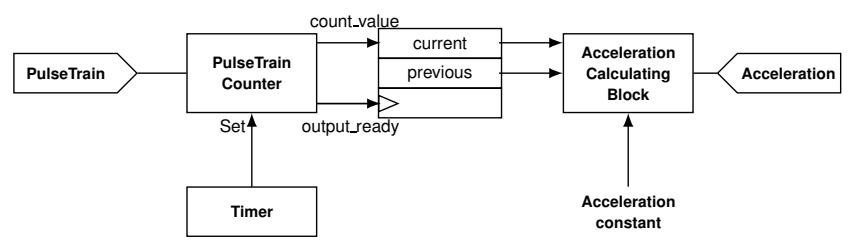

Fig. 9. The acceleration calculator.

\section{CONTROL LoOP}

Due to the hardware flexibility, building a customized controller is straightforward. In general, the complete motor control schematic is shown in Fig. 10, Two alternative control loops are implemented in the current setup, a position based velocity P-controller and a position based PI-controller. The control loop output is converted to a 12-bit binary value proportional to the PWM duty cycle, plus an offset to produce a lock-antiphase PWM signal.

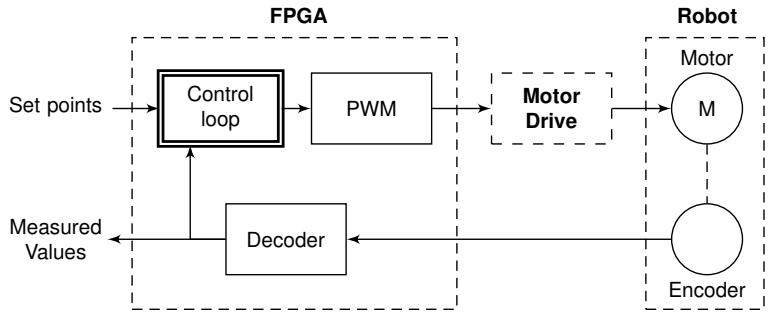

Fig. 10. The complete motor control schematic.

1) Position based velocity P-controller: In the position based velocity P-controller, the difference between desired and measured positions is amplified by a proportional gain $K p$. The measured velocity tunes the amplified signal to generate a trapezoidal velocity trajectory. The control loop is shown in Fig. 11.

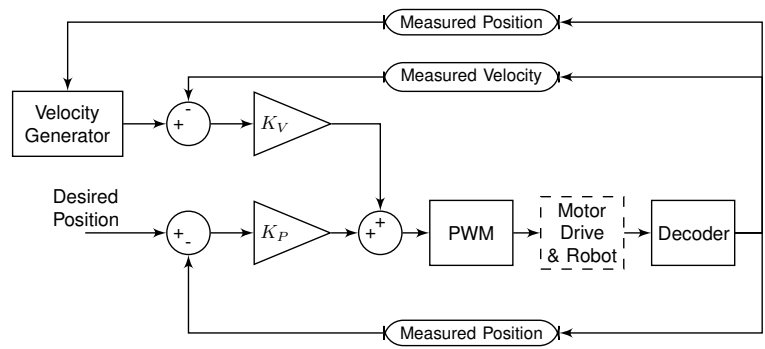

Fig. 11. Position based velocity control loop. The desired trapezoidal velocity trajectory is calculated by the current position of the axis.

2) Position based PI-controller: The steady state error of P-controllers leads to a non-negligible difference between the desired and reached positions. When the desired position is very close to the current, the output control signal becomes too small to drive the motor. A PI-controller with integration clamping [19] compensates the steady state error, as shown in Fig. 12,

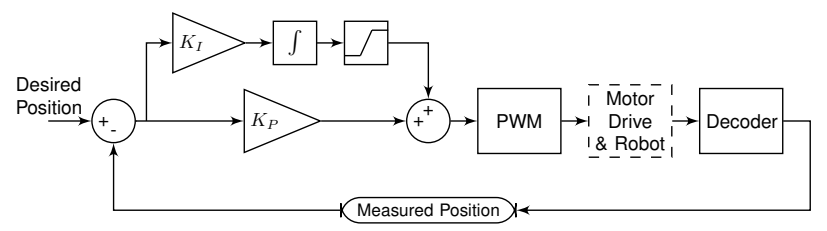

Fig. 12. A PI-controller with integration saturation can eliminate the steady state error.

\section{EXPERIMENT}

The position based PI controller presented in the control loop block in Fig. 10 is wrapped in an IP-core. To verify the hardware control loop performance, as well as to reveal the advantage of hardware co-processing, $\mathrm{C}$ programmed software velocity calculator and PI control loop are tested on the PowerPC processor. 


\section{A. Time-based position trajectory}

A sinusoidal position trajectory in function of time is tested on both hardware and software control loops applied on the wrist axis of the Performer MK2 robot arm:

$$
p_{\text {desired }}=\sin (2 \pi k t)
$$

where the desired position $p_{\text {desired }}$ is calculated as a function of elapsed time $t$ and frequency $k$.

The sine function determined that the reachable angular position varies in a range of $[-1,1]$; the moving direction changes every $\frac{1}{2 k}$ second. However, in practice, the maximum velocity of a motor is limited. Therefore, the reachable angular position range is reduced due to shorter acceleration and deceleration time caused by higher $k$. Fig. 13 compares the stability of the hardware and software control loops, with $k=3,20$ and $40 \mathrm{~Hz}$ respectively.The hardware control loop sustains stable in a high frequency $k=40 \mathrm{~Hz}$, while the software control loop seriously loses its stability. Obviously, driving a multi-axis robot by a software control loop will negatively influence the control performance, as a consequence of introducing multiple function calls.

\section{B. Velocity calculation in software}

The software PI control loop receives measured positions from the decoder. The velocity is calculated in software by:

$$
V_{\text {sw }}=\frac{p_{\text {current }}-p_{\text {previous }}}{t_{\text {elapsed }}}
$$

where $p_{\text {current }}$ denotes the current sampled position, $p_{\text {previous }}$ denotes the previous sample and $t_{\text {elapsed }}$ implies
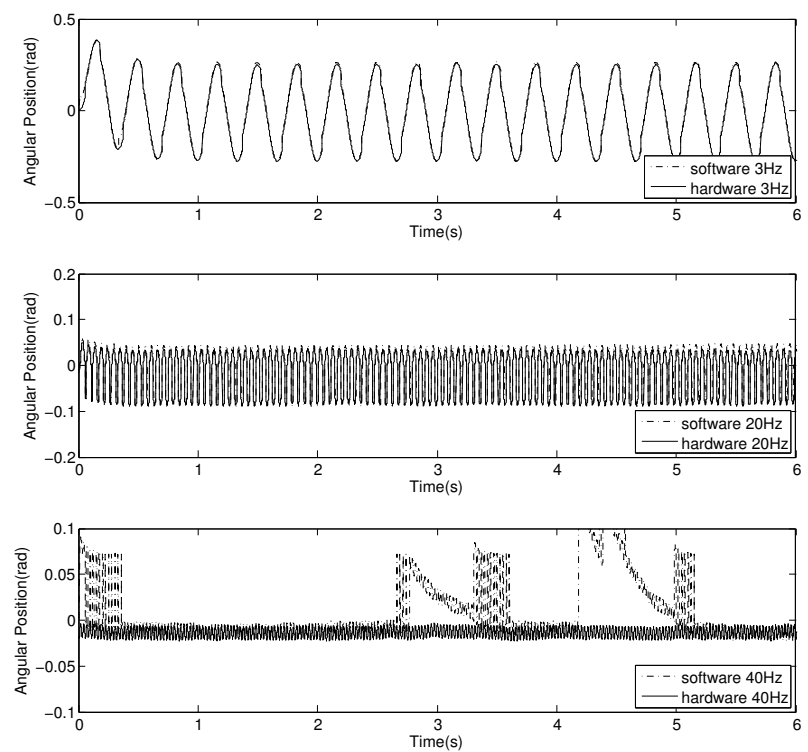

Fig. 13. A sinusoidal position trajectory in function of time is tested on both hardware and software control loops. The hardware control loop has a better performance on high frequency direction change than the software control loop. the time difference between the two samples. The software calculated velocity is noisy because the measurement noise is amplified by the time derivative. Therefore, an $n$-sample moving average is applied on the calculated velocity to filter out the high frequency spikes. Fig. 14 demonstrates the result of applied moving average filters with $n=8,16,32$, 64, 128 and 256 respectively comparing with the hardware calculated velocity. Moving average filter reduces the high frequency noise with a higher $n$; however, as a trade off, it introduces a larger delay while $n$ increases. The hardware velocity calculator based on the pulse train is more reliable compared to the one in software.

\section{Discussion, CONCLUSION AND FutURE WORK}

\section{A. Decimal representation and operations}

Xilinx ISE 10.1 provides floating point operation IPcores compliant with IEEE-754 representation. However, this representation is not applicable for the current setup, due to two factors: the limited resolution of the incremental optical encoder on the robot, and high consumption of configurable logic blocks (CLB) for floating point operation. Each control loop with floating point operation exhausts approximately $22 \%$ of the CLBs on the Virtex-II pro board; however, a complete setup requires five control loops and other peripheral supports, which implies insufficient resources.

To address the resource exhaustion problem, while keeping an adequate precision for decimal calculation, fixed point representation is applied in the current setup. The adapted non-standard fixed point representation requires only $10 \%$ of the CLBs for each axis controller, negatively resulting in extra conversions in software to interpret the fixed point data. However, the significant saving on CLBs permits building complex control loops, or extending robot functions, e.g. visual servoing which utilizes $97 \%$ of the CLBs, while retaining all functions on a single FPGA board with low power consumption of approximately 8 Watt.

\section{B. Flexibility and reliability}

This paper proposes an open embedded hardware and software architecture for industrial robot control. The proposed architecture is applicable to any type of robot, as long as the motors are equipped with incremental position encoders and driven by PWM signals. This architecture has been tested on an industrial Performer MK2 robot. The controllers running on a Xilinx FPGA Virtex-II pro board with embedded Linux provides a flexible platform compared with regular PC-based controllers. Due to the reconfigurability of the FPGA, the functionality can be rebuilt or extended almost immediately. Moreover, the various IOs integrated on the FPGA board properly fits sensor fusion applications. In addition, the IPcores in VHDL are configuration independent and therefore fully reusable. 

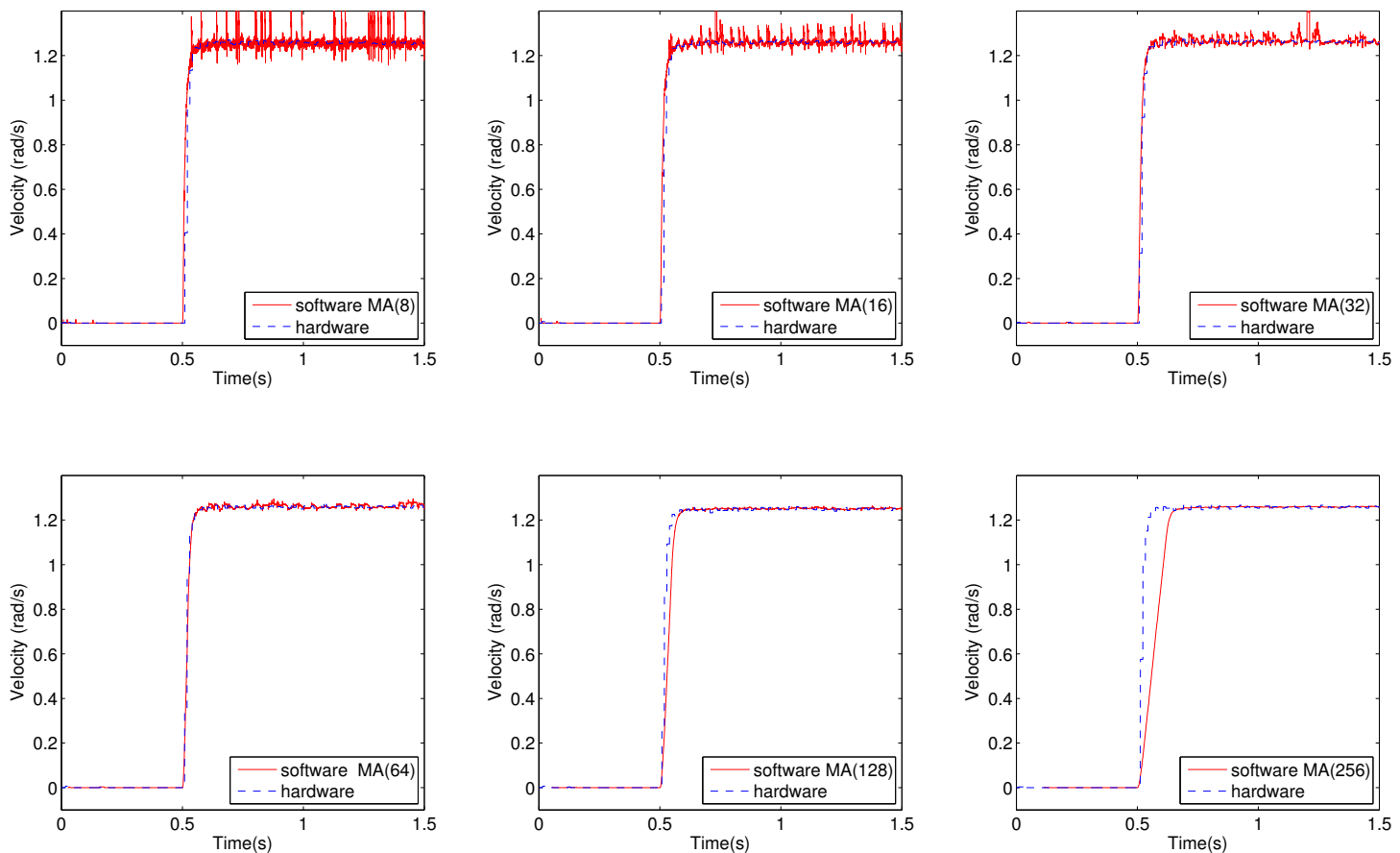

Fig. 14. The result of applied moving average filters with $n=8,16,32,64,128$ and 256 respectively, comparing with the hardware calculated velocity

In the hardware design, the IP-cores are driven by a dedicated clock, which is independent of software and operating system activities running on the PowerPC processor. As demonstrated in the experiment result, the controller, behaving as a co-processor, relieves the PowerPC processor computation load and improves the calculation and stability. In addition, being built on the FPGA, the controller is immunity to any unexpected software crash. The OROCOS software guarantees a robust real-time platform for predictable behaviours, and potentiates embedded robot interaction.

Low cost, low power consumption, remarkable stability, high flexibility and expandability are the key advantages of the proposed architecture for industrial robot control.

\section{Future work}

A heterogeneous hardware coprocessing architecture for embedded robot application is being developed as a follow up of this paper. The hardware, including FPGA and GPU, provide a considerable flexibility on robot control and vision algorithm processing. The integration of various parallel computing hardware creates a remarkable opportunity for low cost and high performance robot interaction.

An advanced and configurable controller library in robot control context is being built with impedance control, full arm dynamics and time-optimal MPC. The creation of this library is assisted by a multi-hardware co-design tool developed by the authors, aiming at bringing OROCOS level computations, i.e., more complex control and estimation, to the hardware level.

A visual servoing application is implemented on the current setup with Kinematics and Dynamics Library (KDL) [20] to verify the real-time performance of the proposed architecture as shown in Fig. 15.

A monochrome XC-55BB camera is mounted on the end effector of the robot, capturing 720 by 480 pixels gray scale frames. The captured frames are processed by a real-time

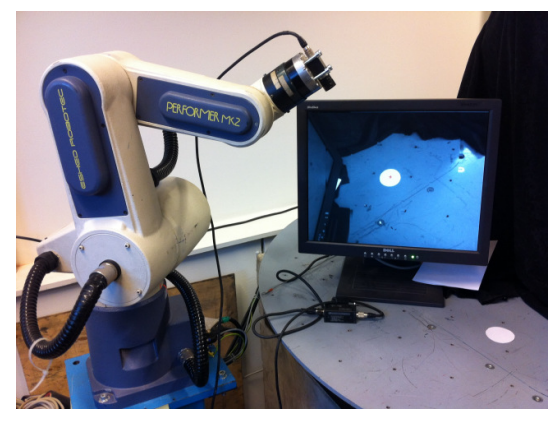

Fig. 15. A visual servoing application based on the proposed architecture is implemented on the current setup. 
blob tracking algorithm implemented in VHDL, which reports the center coordinates of the detected blob in the image. The robot, controlled by an OROCOS program running on the embedded Linux system, moves its end effector in realtime of $30 \mathrm{~Hz}$ to keep the detected blob in the center of the captured image. A performance evaluation of this application is currently being processed.

The IP-core of the PI-controller will be available on http://people.mech.kuleuven.be/ lin.zhang as soon as the paper is published.

\section{ACKNOWLEDGEMENT}

The authors gratefully acknowledge the financial support by KU Leuven's Concerted Research Action GOA/2010/011 Global real-time optimal control of autonomous robots and mechatronic systems.

\section{REFERENCES}

[1] H. Bruyninckx, "Open RObot COntrol Software," http://www.orocos.org/ 2001, last visited June 2011.

[2] M. Prats, S. Wieland, T. Asfour, A. P. del Pobil, and R. Dillmann, "Compliant interaction in household environments by the Armar-III humanoid robot," in IEEE-RAS International Conference on Humanoid Robots, 2008, pp. 475-480.

[3] C. Carozzi, G. Magnani, and S. Nicolodi, "Implementation of sensorbased control algorithms in an industrial robot controller," Control Engineering Practice, vol. 3, no. 9, pp. 1307-1313, 1995.

[4] J. S. Gu and C. W. de Silva, "Development and implementation of a real-time open-architecture control system for industrial robot systems," Engineering Applications of Artificial Intelligence, vol. 17, no. 5, pp. 469-483, 2004.

[5] H. Le-Huy, "Microprocessors and digital ics for motion control," Proceedings of the IEEE, vol. 82, no. 8, pp. 1140 -1163, aug 1994.

[6] T. N. Chang, B. Cheng, and P. Sriwilaijaroen, "Motion control firmware for high-speed robotic systems," IEEE Transactions on Industrial Electronics, vol. 53, no. 5, pp. 1713-1722, 2006.

[7] E. Monmasson and M. N. Cirstea, "FPGA design methodology for industrial control systems-A review," IEEE Transactions on Industrial Electronics, vol. 54, no. 4, pp. 1824-1842, 2007.

[8] B. Berkeley Design Technology Inc, "Comparing fpgas and dsps for high-performance dsp applications," 2006.

[9] H. Liu, P. Meusel, N. Seitz, B. Willberg, G. Hirzinger, M. H. Jin, Y. Liu, R. Wei, and Z. W. Xie, "The modular multisensory DLR-HIThand," Mechanism and Machine Theory, vol. 42, no. 5, pp. 612-625, 2007.

[10] R. Dubey, P. A. Agarwal, and M. Vasantha, "Programmable logic devices for motion control - a review," IEEE Transactions on Industrial Electronics, vol. 54, pp. 559-566, 2007.

[11] E. Galvan, A. Torralba, and L. G. Franquelo, "ASIC implementation of a digital tachometer with high precision in a wide speed range," ITIE, vol. 43, no. 6, pp. 655-660, 1996.

[12] B. Hebert, M. Brule, and L.-A. Dessaint, "A high efficiency interface for a biphase incremental encoder with error detection [servomotor control]," IEEE Transactions on Industrial Electronics, vol. 40, no. 1, pp. $155-156,1993$.

[13] ABB, "ABB Robotics," http://www.abb.com/robotics/ 2011.

[14] KUKA, "KUKA n.v." http://www.kuka.be/ 2007.

[15] B. Gerkey, R. Vaughan, A. Howard, and N. Koenig, "The Player/Stage project," http://playerstage.sourceforge.net/ 2001.

[16] M. S. Arefeen, D. Figoli, and Z. Yu, "Integrating multiple motor control functions using a single DSP controller," in Applied Power Electronics Conference and Exposition, 1999. APEC '99. Fourteenth Annual, vol. 2, mar 1999, pp. 813-818 vol.2.
[17] F. Garcia, E. Diez, and F. L., "Design of a high precision digital tachometer for speed and position measurement," in Design of Integrated Circuits and Systems DCIS 2000, vol. 15, Montpellier,France, 2000, pp. 1-9.

[18] M. Bonert, "Digital tachometer with fast dynamic response implemented by microprocessor," IEEE Transactions on Industrial Applications, vol. IA-19, pp. 1052-1056, 1983.

[19] A. S. Hodel and C. E. Hall, "Variable-structure pid control to prevent integrator windup," IEEE Transactions on Industrial Electronics, pp. 442-451, 2001.

[20] R. Smits, "KDL: Kinematics and Dynamics Library," http://www.orocos.org/kdl 2001, last visited 2011. 